\title{
The use of network analysis to creating a model of Triangulated Irregular Network (TIN) on the basis of the data from Polish Active Geodetic Network EUPOS (ASG EUPOS) stations
}

\author{
Anna Maria Kowalczyk, Kamil Kowalczyk \\ University of Warmia nad Mazury, Oczapowskiego 2, 10-900 Olsztyn, Poland
}

\begin{abstract}
A system of referential stations ASG EUPOS launched in 2008 in Poland provides, among others, information regarding the changes of the stations' vertical and horizontal coordinates. These changes can be related to the changes of tectonic plates. One can create models of the vertical and horizontal movements of the Earth's crust. The changes of height of the referential stations are more difficult to model than horizontal changes. The evaluation of credibility of these changes is also more challenging. The velocities of the vertical changes can be determined in two ways: as absolute velocities (on referential stations) or as relative velocities - between the stations. Although the referential stations are located evenly within the Polish territory, they do not compose preordained geometric shapes. Such an arrangement allows for certain freedom of creating connections of the stations between which the relative vertical movements are determined. Although one popular method of creating the TIN is Delaunay triangulation, there are methods relating to network analyses allowing for connecting the points in another way. The purpose of this article is to employ various analytical methods to create the TIN of the relative vertical movements. The chosen methods of network modelling are characterised and compared. Moreover, their utility regarding the creation of a model of relative vertical movements within Poland's territory is evaluated.
\end{abstract}

Keywords: network analysis; model; vertical movements; ASG EUPOS.

\section{Introduction}

A wide range of interpolation methods are used to build spatial models connected with physical phenomena. Usually, primary data is not placed regularly and the choice of joint is related to the interpolation method used. Through the choice of the interpolation method, we can limit for example the influence of phenomena taking place within greater distances, as well as through the choice of the most advantageous adjoining points. With that choice, TIN -a network of triangles is built. If we assume a linear character of a change in a certain phenomenon, it is then indispensable to delineate the joints between which interpolation will be conducted. Not knowing the dependencies between the values obtained in adjoining measure points, we use assumption and mathematical solutions which define these joints. The most widespread method is Delaney's triangulation [7]. There are, however, methods which do not base on triangulation. In these methods, joining is done via determining mutual relations between adjoining values and seeking a course of similar characteristics [3]. It can be said that they form specific skeletal lines in surface models of physical phenomena.

During triangulation, a network is created - a certain number of connections. The arrangement of joints in this network is random, characterising with Poison's normal arrangement. In random networks, each node has thereabout a similar number of joints. In the 1990s, it was discovered [5] that there are networks with a power arrangement. These, known as scale-free networks, are characteristic for, among others, the existence of cents (hubs) - nodes with a far greater number of joints in comparison to the others, the ellimination of which results in substantial damages in the network's structure or in visible disturbances of functionality. Moreover, we can distinguish modular networks, hierarchical networks (they may also have the characteristisc of scale-free networks) and small world network [4]. Constructing and analysing of network models, as well as stating their character allows for using their properties and is a tool for optimising decisions. We can find a lot of examples in literature $[4,5,6,8]$ concerning working out network models in different areas of life. From brain cells, neurons, communication networks to social relations, everything could be presented and analysed in the aspect of network.

Corresponding author: Kamil Kowalczyk. E-mail address: kamil.kowalczyk@uwm.edu.pl

http://dx.doi.org/10.3846/enviro.2014.220

(C) 2014 The Authors. Published by VGTU Press. This is an open-access article distributed under the terms of the Creative Commons Attribution License, which permits unrestricted use, distribution, and reproduction in any medium, provided the original author and source are credited. 
Networks are arrangements of nodes and joints between them. The connections can be physical (for example roads and cities) or they can concern certain dependencies (for example the number of quotations for a scientist) [4]. We can distinguish six possible ways of linking any values [3]:

- connecting values by the course of minimal increase in value (Fig. 1);

- connecting values by the course of maximum increase in value (Fig. 2);

- connecting values by the course of minimal decrease in value;

- connecting values by the course of maximum decrease in value;

- connecting values by the course of minimal differences in value;

- connecting values by the course of maximum differences in value.

(a)

\begin{tabular}{|c|c|c|c|c|c|}
\hline \begin{tabular}{l|l}
23 & 1
\end{tabular} & 18 & \begin{tabular}{l|l}
13 & 5
\end{tabular} & \begin{tabular}{l|l}
5 & 15
\end{tabular} & 27 & \\
\hline 30 & 11 & 22 & 8 & 6 & 24 \\
\hline \begin{tabular}{l|l}
25 & 1
\end{tabular} & 19 & \begin{tabular}{l|l}
4 & 10
\end{tabular} & \begin{tabular}{l|l}
0 & 16
\end{tabular} & 14 & \\
\hline 12 & & 28 & & & 20 \\
\hline 26 & & \begin{tabular}{l|l}
77 & 21 \\
\end{tabular} & \begin{tabular}{l|l}
1 & 12 \\
\end{tabular} & & \\
\hline
\end{tabular}

(b)

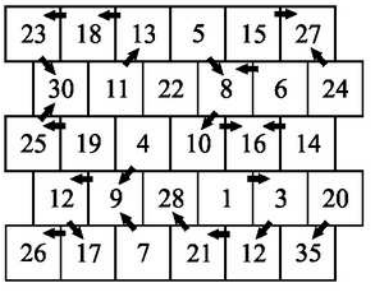

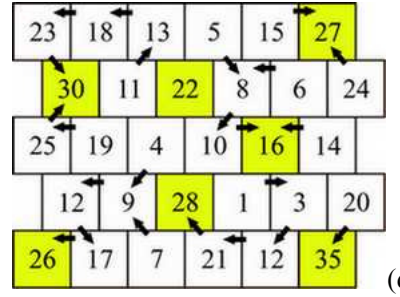

(d)

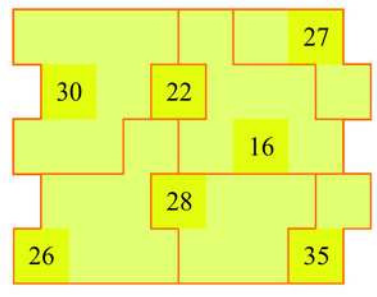

Fig. 1. Connecting values by the course of minimal increase - connections are possible only to neighbour fields

\begin{tabular}{|c|c|c|c|c|c|c|}
\hline 23 & 18 & 13 & 5 & 15 & 27 & \\
\hline 36 & $\begin{array}{lll}0 & 1\end{array}$ & 18 & 22 & 8 & & 24 \\
\hline 25 & 19 & 4 & 10 & 16 & 14 & \\
\hline & 29 & 9 & 28 & 1 & 3 & 20 \\
\hline 26 & 17 & 7 & 21 & 12 & 3 & \\
\hline
\end{tabular}
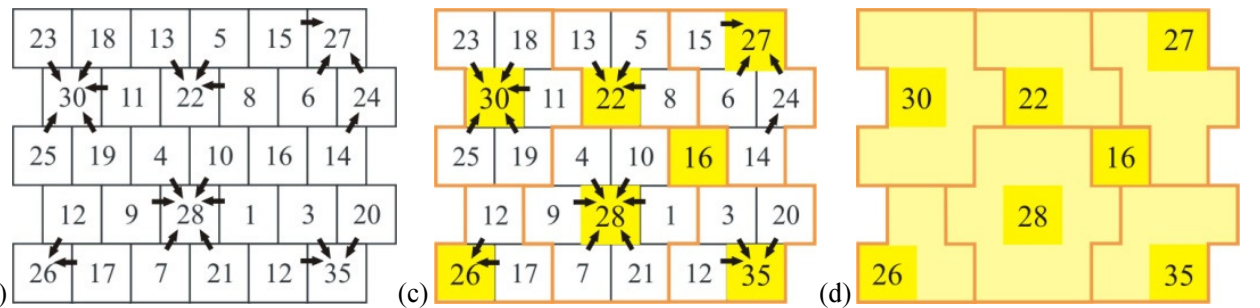

Fig. 2. Connecting values by the course of maximum increase - connections are possible only to neighbour fields

The above models present the process of forming a network and the spatial diversity of the organisation of this phenomenon. It may seem that some of the rules are identical, yet, analysing the above theoretical examples, we can see that it is not so. In the presented examples of the connection values it has been assumed that a certain area is represented by basic fields; their centres have been ascribed (randomly) with certain spatial features representing a certain sphere differentiating geographical space. These features may represent any spatial values which describe the phenomena generating its development or any change of its condition.

The issue of spatial connections, created on the basis of the suggested rules, came to life during the research on the planification space use, at different levels of its organisation [1,2]. It has also been pointed out [3] that there is a connection between the suggested spatial links with the theory of spatial self-organisation. It has been stated that there is a corelation between spatial self-organisation and the formation of spatial relations network. It is assumed that the self-organisation must proceed according to some rules, constituting an immanent characteristic of a geographical space. "The notion of "organisation" itself suggests a need to arrange and the prefix "self" suggests that feedback should be the basic mechanism of self-organisation' [3].

Vertical movements of the crust is a physical phenomenon influenced by various factors. They can be elaborated on the basis of different data and different network constructions. Until now, in Poland, vertical movements models heve been elaborated on the basis of levelling data [9]. A levelling network is a network where the curves are measured by a set course. It is different when we wish to determine vertical movements on the basis of satellite data measured on permnent stations. The stations are not connected with a course but with a vector.

The following article describes an attempt to use network analyses for orgnising a geographical space with relation to physical phenomena. As data, we can find unlevelled linear trends on delineated on vectors between the stations of the ASG EUPOS network. The trends were calculated from daily solutions of the differences in heights between the stationd distanced from one another not more than $70 \mathrm{~km}$. For elaboration, for cognitive purposes, also vectors to the chosen premanent stations of the neighbouring countries were included.

In the process of constructing the network model, as well as in further analyses, stress was put on the following two cases of connecting values:

- by the course of minimal increase;

- by the course of maximum increase.

The nodes in these networks stand for the reference stations and the joints are the spatial dependencies of minimal or maximum increase between the particulr nodes (unlevelled linear trend). The obtained results were presented in the pictures, described and compared with the calculated values of un-closing of particular eyelets, as well as with the values of the vertical movment in the nodes obtained by the initial network levelling. 


\section{Nodes connections}

The analysis of the networks in the two above-mentioned variants is supposed to show the possible interactions between particular connections and the extent of their activity. The models elaborated on that basis also present the flow of values how a particular value reaches particular terminal nodes and by what course that is conducted. A double-track approach allows us to state which method of network modelling is optimal for our purposes and to tell which nodes the points of the network are leading to. The suggested methods also allow us to verify the correctness of the data undergoing analysis. In both analyses 123 nodes and 344 connections were taken into consideration.

\subsection{Connecting by the course of minimal increase}

In the first stage of the analysis a method of connecting by the course of minimal increase was used. The results were displayed in (Fig. 3). The resulting joints do not form a big, concise network, they form networks of several nodes. The nodes which the joined values were aiming at, were marked with a circle. Thirteen such nodes were noted. According to the above theory, these nodes constitute points of identical activity on the adjacent vectors. The biggest network (26 joints) was observed near the Eastern border of Poland. In two cases, values converged into peripheral. This is an anomaly considering the theoretical assumption of this method. It may prove an error in the prepared data. The settled linear trends were examined and no errors were stated. One should remember that the trend values which were elaborated are unlevelled, so there is a possibility of errors in the measurement data itself (for example considerable noise).

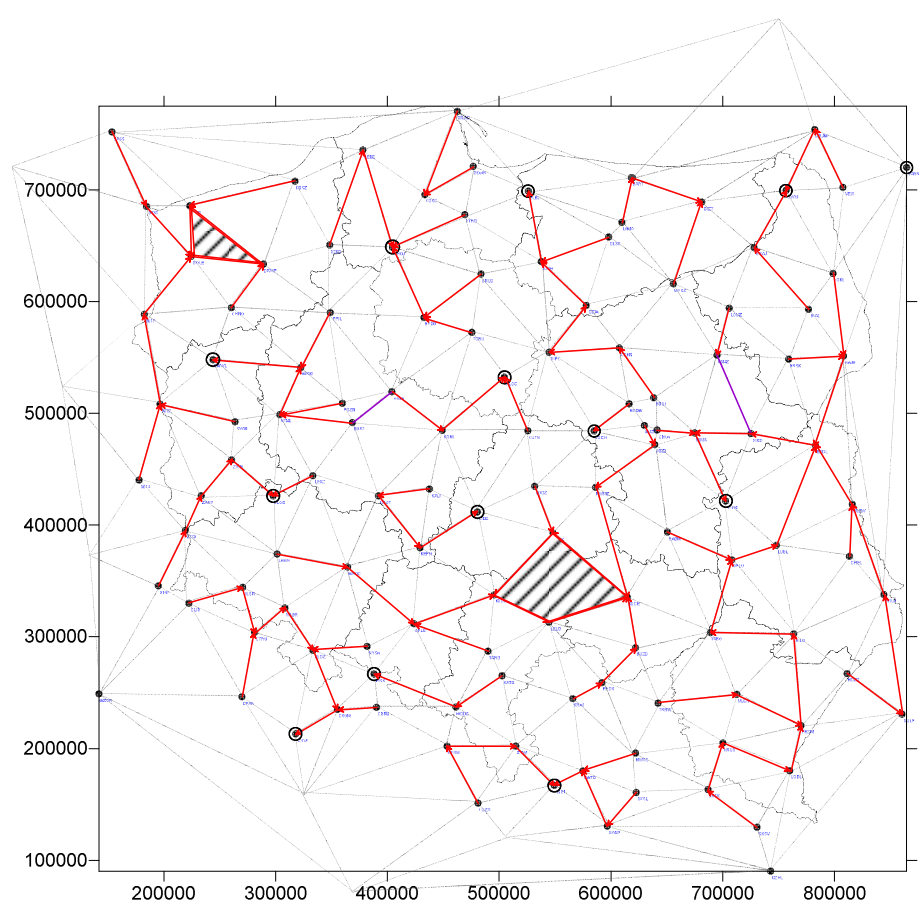

Fig. 3. Grey lines - triangulation network. Red lines - the network of connecting by the course of minimal increase in value. Black points - nodes. The biggest black points- thirteen nodes which attract the others. Texture - a circle connections

\subsection{Connecting by course of maximum increase}

In the second stage of the analysis, a method of connecting by course of maximum increase was used. The results were displayed in (Fig. 4). The resulting joints do not form a big, concise network, they form quite regular stars. As above, the nodes which the values were aiming at, were marked with a circle. Thirteen such nodes were noted. There is no point with an advantage of the adjoined values. In three cases, values converged into peripheral. This can be explained as in the firs analysis. In one case, the anomaly area re-ocurred.

\section{Detailed analysis}

The results of the analysis were confronted with the calculations of un-closing of triangles and with the initial results of network levelling to the vertical movements from the satellite data. Levelling was conducted assuming a stable point in central Poland. 


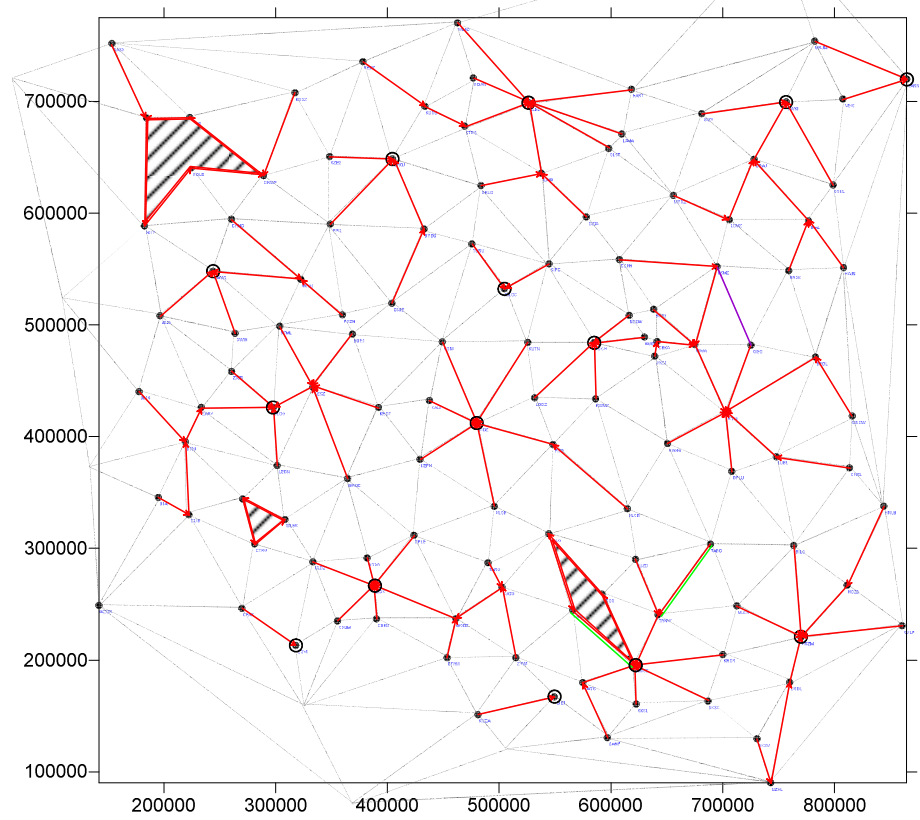

Fig. 4. Grey lines - triangulation network. Red lines - the network of connecting by the course of maximum increase in value. Black points - nodes The biggest black points- thirteen nodes which attract the others. Texture - a circle connections



Fig. 5. Grey lines - triangulation network. Red lines - the network of connecting by the course of minimal increase in value. Black points - nodes. Texture - a circle connections. Circle with black fill/ without $--/+$ misclosure of loops

\subsection{Variant 1}

Comparison of variant 1 (minimal value increase) with misclosure of loops is presented in (Fig. 5). No coincidence was observed between the scale of un-closing and the connection of values by course of minimal increase.

Another verification was examining the dependencies between variant 1 and relative vertical movements in premanent stations. For a better picturing of the phenomenon, a linear interpolation was conducted (Fig. 6). In seven cases, the main nodes of attraction agree with local extremes from the interpolation. The course of isolines cuts the elaborated skeletal lines in most parts. 


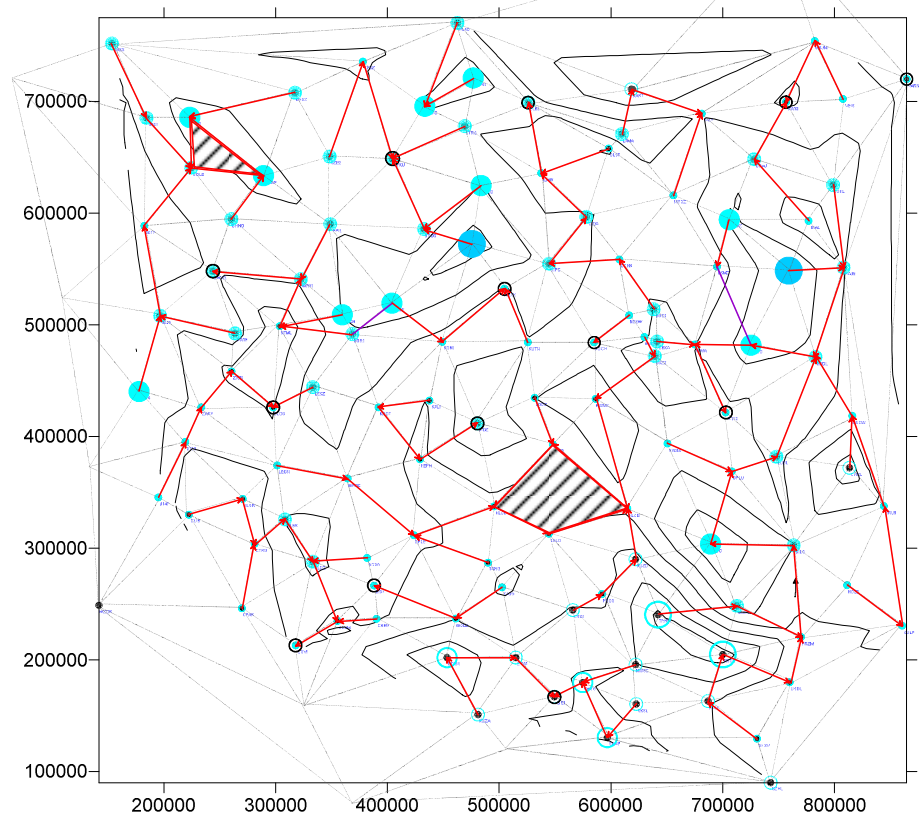

Fig. 6. Grey lines - triangulation network. Red lines - the network of connecting by the course of minimal increase in value. Black points - nodes which attract the others. Texture - a circle connections. Black lines - izolines. Circle with blue fill/ without $--/+$ relative crustal movements

\subsection{Variant 2}

Comparison of variant 2 (maximum value increase) with misclosure of loops is presented in (Fig. 7). Unlike in variant 2 , some coincidence was observed between the scale of misclosure of loops and the connection of values by course of maximum increase. In three of five cases (the biggest misclosure of loops), the resulting anomalies agree with the big misclosure of loops. This can encourage further research on this variant in order to learn more about these dependencies (for example ellimination only of the joints pointed to in the analysis from the levelling process).

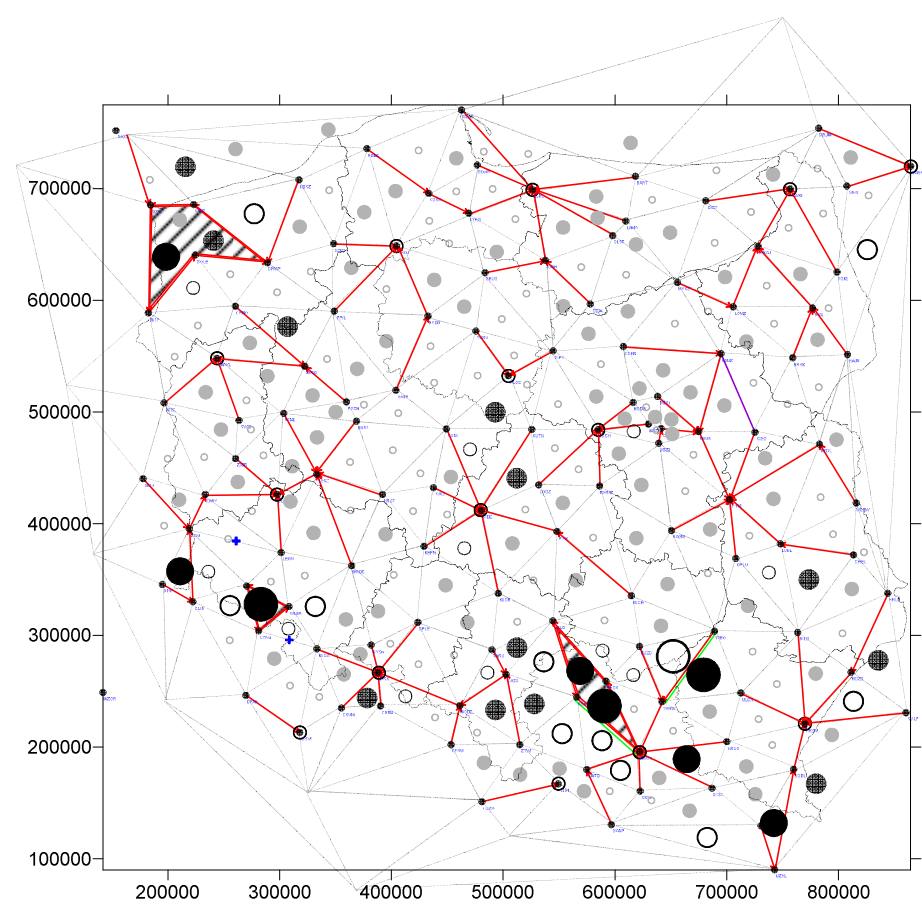

Fig. 7. Grey lines - triangulation network. Red lines - the network of connecting by the course of maximal increase in value. Black points - nodes Texture - a circle connections. Circle with black fill/ without - -/+ misclosure of loops

Another verification was examining the dependencies between variant 2 and relative vertical movements in premanent stations. The result is displayed in (Fig. 8). In seven cases the main nodes of attraction agree with local extremes from the interpolation. The course of isolines cuts the elaborated skeletal lines in most parts. 


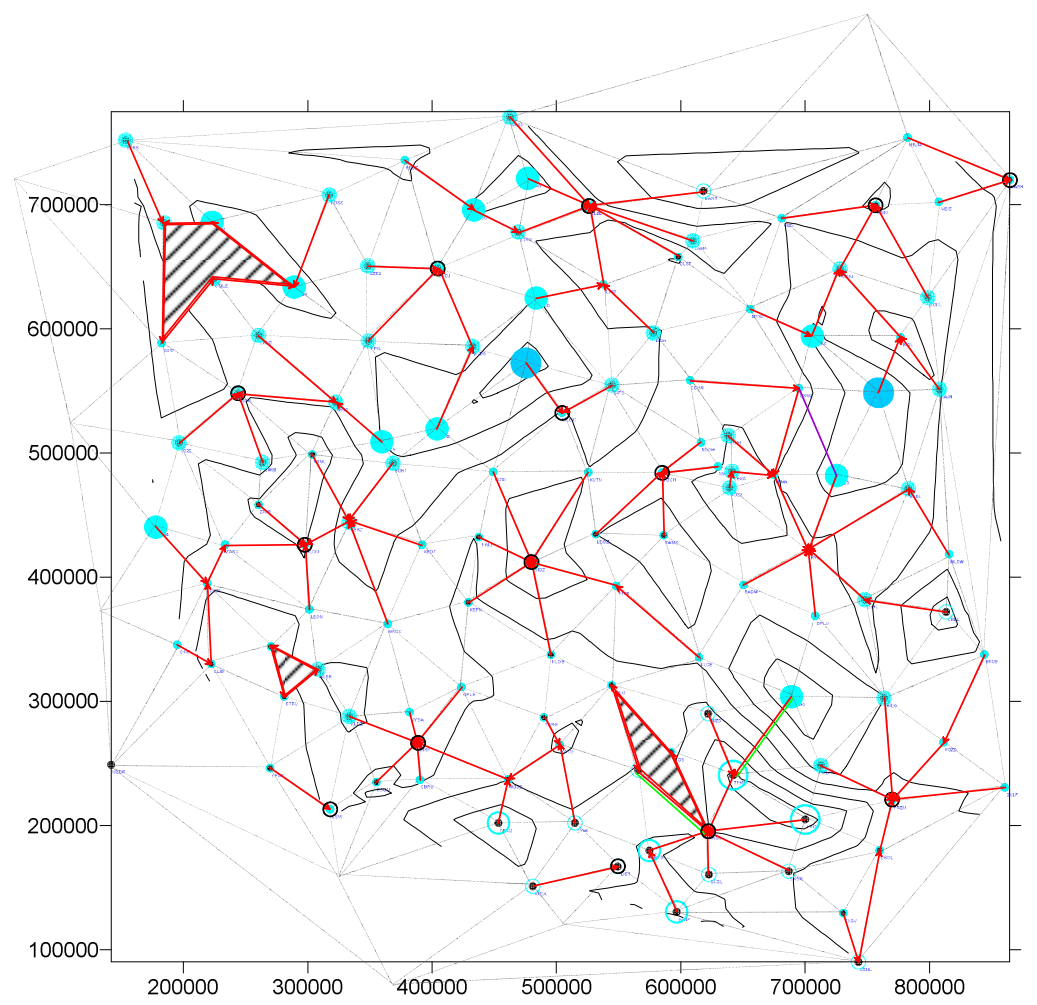

Fig. 8. Grey lines - triangulation network. Red lines - the network of connecting by the course of maximum increase in value. Black points - nodes which attract the others. Texture - a circle connections. Black lines - izolines. Circle with blue fill/ without $--/+$ relative crustal movements

\section{Summary}

The conducted analyses give us a view on the possibilities of using network analyses to verify or construct a vertical movements model. Two variants out of six possible were analysed. In both cases some dependencies between the selected aggregation points and the course of isolines were discovered. A deta ansledwer to the question, though, requires a thorough analysis, together with re-levelling of the network - with ellimination of systematic errors and connections with substantial amendments. The comparison of the misclosure of loops revealed a certain coincidence with the maximum value increase method. In both variants of analysis, 12 selfsame nodes were appointed. They have a massive influence on the value of movements in the region. It can be especially important in the process of network levelling or of seeking common points for simultaneous levelling of the vertical movements model from the levelling and satellite data. In both variants, regional aggregation points are formed which attract several joints each. Variant 1 is an exception - a point attracting 26 joints was noted.

The resulting joints bear traits of skeletal lines. This property can be used to verify the course of isolines, assuming that they should cut these connections. On the basis of the conducted analyses, we could not exclude the usefulness of the examined methods in the process of constructing a vertical movements model. Further teste should, however, be conducted, using different combination of data and the remaining four network analyses methods.

If we assume the rightness of the theory, the models should be improved and the elaborated skeletal lines should influence the choice of weight. The task should be repeated after elliminating systematic errors from the material and after examining and possibly elliminating the stations which are burdened with massive trend errors, as well as the connections which obtained maximum amendments in the process of levelling.

\section{References}

[1] Bajerowski, T. 2003. Podstawy teoretyczne gospodarki przestrzennej i zarzqdzania przestrzeniq. Wydawnictwo Uniwersytetu WarmińskoMazurskiego, Olsztyn.

[2] Bajerowski, T. 2003. Niepewność w dynamicznych układach przestrzennych. Wydawnictwo Uniwersytetu Warmińsko-Mazurskiego, Olsztyn.

[3] Bajerowski, T.; Biłozor, A. 2005. Theory of Barabasi scale-free networks as a new tool in researching the structure and dynamics of regions, Regional scientists' tribute to professor Ryszard Domański., Studia Regionalia 15: 209-230. ISSN 0860-3375.

[4] Barabási, A.-L. 2003. Linked. Penguin Group.

[5] Barabási, A.-L.; Bonabeau, E. 2003. Scale-Free Networks, Scientific American, 50-59.

[6] Barabási, A.-L.; Réka, A.; Hawoong, L. 2000. Scale-free characteristics of random networks: the topology of the world-wide web, Physica A 281, 6977. http://dx.doi.org/10.1016/S0378-4371(00)00018-2

[7] Delaunay triangulation. 2010. Wolfram MathWorld.

[8] Kowalczyk, A. 2012. The iconic model of landscape aesthetic value, European Spatial Research and Policy. 2/2012, 121-128.

[9] Kowalczyk, K. 2005. Determination of land uplift in the area of Poland, in $6^{\text {th }}$ International Conference Environmental Engineering. 1-2: 903-907. 